\title{
Radiation induced angiosarcoma of the hypopharynx: a rare case report and review of literature
}

\begin{abstract}
Radiation-induced sarcoma in the head and neck is a long-term rare complication of radiation therapy, with an estimated risk of up to $0.3 \%$. The incidence of these sarcomas is, however, likely to increase due to the progressive aging of the population combined with improved survival in head and neck cancer patients resulting from better treatment regimes. This is a case of post -radiation sarcoma of the hypopharynx radiated for tongue carcinoma previously. This case is reported to high light a rare but devastating complication of radiotherapy that is to be kept in mind during follow up.
\end{abstract}

Volume II Issue 2 - 2019

\author{
Eyad Darraj, Abdelgalil Ali Ragab, Tarek \\ Salhani \\ Dubai Health Authority, UAE
}

Correspondence: Eyad Darraj, Dubai Health Authority, Dubai, UAE,Tel 0097I52842I23I,Email eyad-ent@hotmail.com

Received: February 17, 2019 | Published: March 21, 2019

\section{Introduction}

Radiotherapy has been considered a standard treatment for malignancies for decades, the need for radiotherapy is estimated to be about $60 \%$ in patients with malignant tumor either for radical treatment or for palliation purposes. ${ }^{1}$ As a result of modern therapies and advances in management protocols, the survival rate of patients with head and neck malignancy has increased, thus more attention now is paid to study the morbidity and side effects of the available treating modalities. ${ }^{2}$ The low incidence of sarcomas makes its diagnosis and treatment a real challenge. ${ }^{3}$ Unfortunately, the ultimate survival rate of sarcoma induced by radiation is lower than primary sarcomas at the same stage. It's thought that the incidence of radiation-induced sarcoma is increasing, this could be a result of old ages along with high success rate of head and neck cancer management. ${ }^{4}$ Although surgical removal of radiation-related sarcoma is the main treatment, ${ }^{5}$ full resection is a big challenge due to the surrounding important structures and multifocal nature of these tumors. What makes the prognosis of incomplete removal much worse is the fact that these tumors are not sensitive to radio or chemotherapy. ${ }^{6}$ In the head and neck area, Squamous Cell Carcinoma is the most commonly encountered histopathologic subtype of radiation- related malignancies, followed by RIS which comprises $12 \%$ of radiation- related cancer. The chance of getting such malignancies ranges between $0.03 \%$ and $0.3 \%$ in those who have a history of radiotherapy. ${ }^{7}$ There is no specific site of origin for the Radio-induced sarcoma, it can originate from any irradiated connective tissue within the head and neck. ${ }^{2}$ Commonly, radio-induced sarcomas resemble anew sarcomas; they could be Fibrosarcoma, chondrosarcoma, osteosarcoma, synovial sarcoma and rhabdomyosarcoma. ${ }^{8}$

Generally speaking, there are no special radiologic characteristics of RIS, and imaging cannot differentiate it from isolated or recurrent sarcomas. On the other hand, the clinician should suspect RIS in case of radiotherapy history with a suitable gap of time along with big size and significant infiltration of the surroundings. ${ }^{9}$ Many factors might play a role in the progression of RIS, such as: the malignant susceptibility of individual genetics, combined therapy with chemical agents, and dose of radiation. Radio-induced sarcoma has been seen with radiotherapy as minute as $15 \mathrm{~Gy}$,. Hence, it's not possible to determine a dose of radiotherapy considered safe. ${ }^{10}$ Surprisingly, an increased dose of radiation is not associated with an increased chance of getting RIC. ${ }^{11}$

A research of children malignancies ended up with a result that: Children who survived cancer have a nine times bigger risk of getting RIC in comparison with others, with maximum possibility in children less than four years old when malignancy was first diagnosed. ${ }^{12}$

\section{Case report}

A 70 years old male patient presented to the emergency department with stridor and dysphagia of 3 months duration. Patient was examined by fiber optic nasopharyngoscope which revealed supra-glottic mass to the left side with restricted mobility of the left vocal cord. Patient was admitted and submitted for urgent tracheostomy. MRI neck with contrast was done and revealed a Supraglottic mass measuring about $2 \mathrm{~cm} \times 1.5 \mathrm{~cm}$, partially occluding the airway and showing enhancement post gadolinium injection. It is appearing isointense in $\mathrm{T} 1$ and $\mathrm{T} 2$-weighted images with mildly enlarged vocal cords (Figure 1-3). Panendoscopy was performed on 18/02/2018 and revealed a mass occupying the post cricoid area, pyriform fossa, supraglottic and the lateral pharyngeal wall. Base of the tongue, tonsils, right side of the larynx and the posterior pharyngeal wall were free.

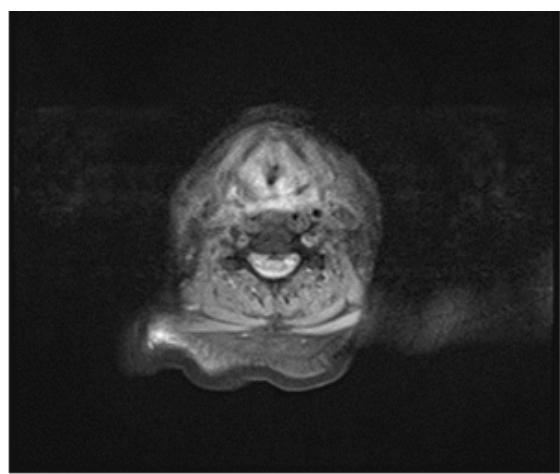

Figure I MRI neck with contrast, axial cut showing supraglottic mass lesion partially occluding the airway.

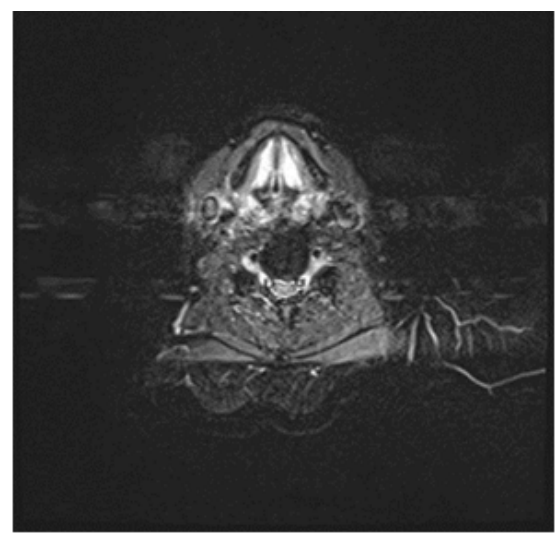

Figure 2 MRI neck, axial cut, showing supraglottic mass lesion partially occluding the airway. 


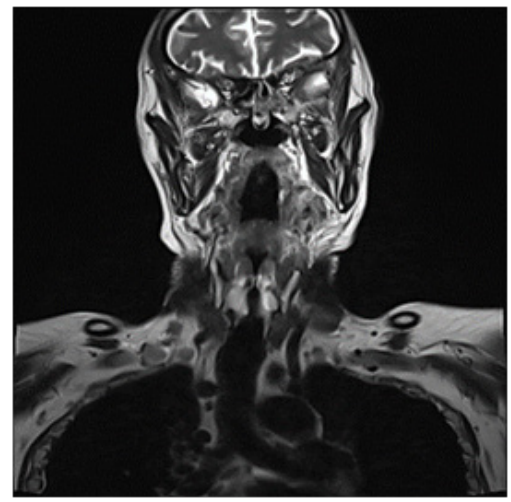

Figure $3 \mathrm{MRI}$ neck with contrast, coronal cut, showing mildly enlarged vocal cords.

A biopsy was taken and histopathology was done using immunohistochemically markers and showed angiosarcoma, PET CT scan was performed on 21/02/2018 and showed the following: hypermetabolic suspicious lymph node in the right infraclavicular region, hypermetabolic focus in the region of the left mandible, adjusting left margin of the tongue and the hypopharynx (Figure 4-6). Patient was diagnosed before as squamous cell carcinoma of the right lateral side of the tongue. Patient was operated in Oman on $8^{\text {th }}$ November, 2006 for a white tongue lesion, and then operated again on 13th December, 2006 for tongue carcinoma. Lastly, partial Glossectomy was done for recurrent tongue carcinoma on 24th March, 2010 (Figure 7). After surgery, patient received total radiotherapy treatment of the face and neck using $6 \mathrm{MV}$ photons, 2 fields and a total dose of 60Gy (44 Gy+20 Gy off cord) from 21st June, 2010 till 03rd August, 2010. Patient was then referred to a specialized center, where further management was done. This case was reported to high light an infrequent long-term complication of radiotherapy.

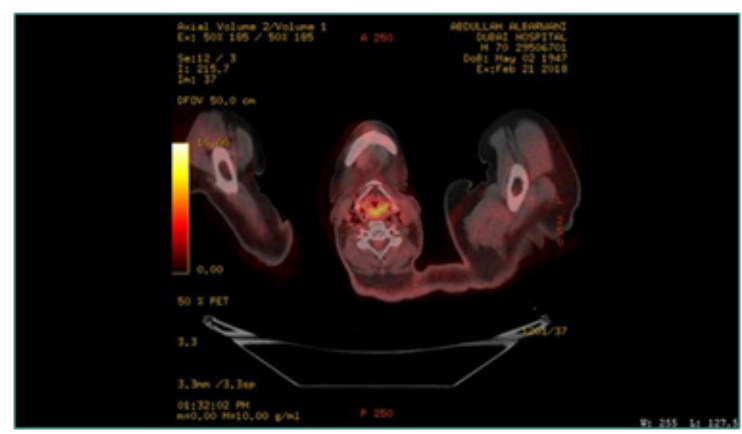

Figure 4 PET CT scan, showing hypermetabolic focus at the hypopharyx, post cricoid area.

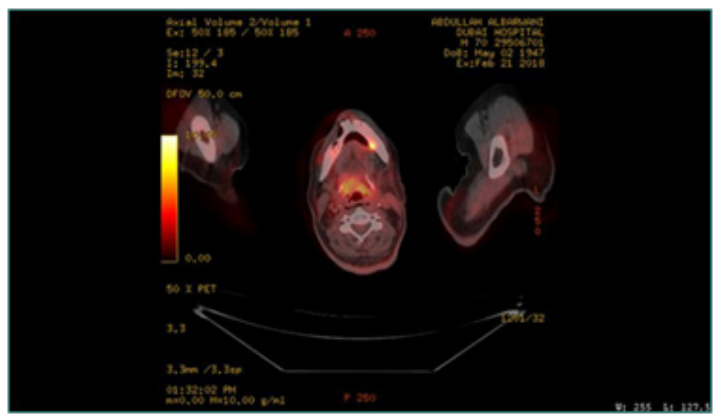

Figure 5 PET CT scan, showing hypermetabolic focus at the region of left mandible, adjusting left margin of the tongue.

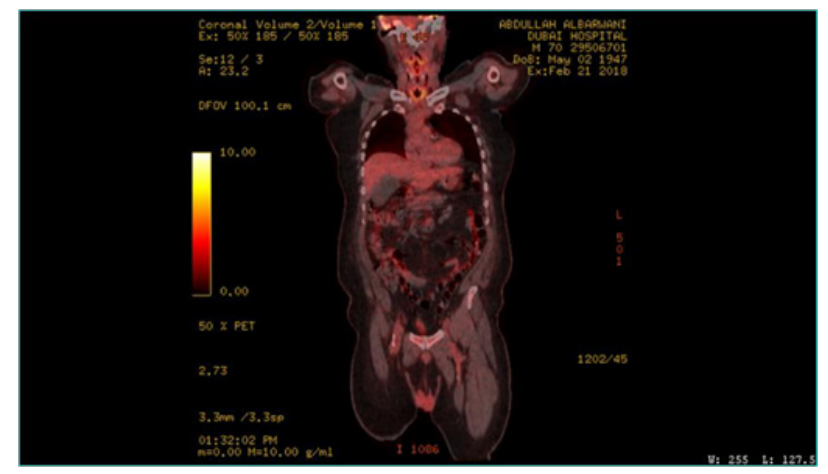

Figure 6 PET CT, showing hypermetabolic suspicious lymph node in the right infraclavicular region, hypermetabolic focus in the region of left mandible.

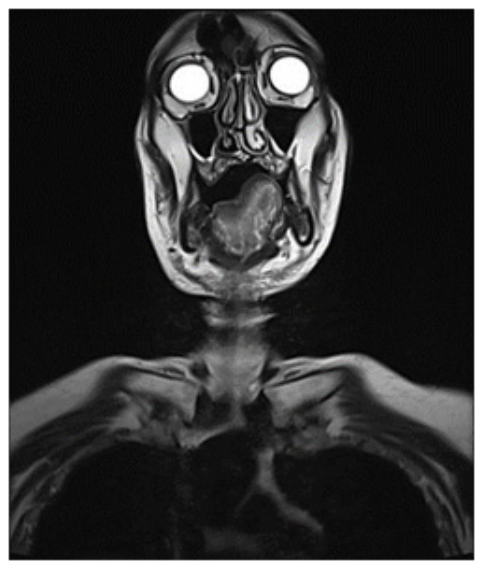

Figure $7 \mathrm{MRI}$ neck, coronal cut, showing the old postoperative changes with right hemi glossectomy.

\section{Discussion}

Sarcomas are not common in the head and neck, they compose $1 \%$ of this region's cancer and no more than $10 \%$ of entire body sarcomas. ${ }^{13}$ While the risk of RIS after radiotherapy is well-known in many anatomical regions, it's less frequently encountered in the head and neck. ${ }^{14}$ Unfortunately these sarcomas are highly malignant, develop many years after irradiation of range of $\mathrm{H} \& \mathrm{~N}$ pathologies. ${ }^{15}$ In this case report, angiosarcoma developed 8years post irradiation. Reviewing literature, it's mentioned that during the period from 1960 until 1989 the frequency of new RIS cases in the H\&N was $0.06 \%$. While this rate increased to $0.17 \%$ in 1990 to $2010 .{ }^{16}$ Although one study reported that sarcoma is the most common radiation induced malignancy in the head and neck followed by squamous cell carcinoma, adenoid cystic carcinoma and poorly differentiated carcinoma. ${ }^{17}$ Other studies ended up that SCC is the commonest cancer post radiotherapy. ${ }^{15}$ Some papers estimate that a range of 8-100Gy could induce sarcoma, and the average dose for malignancy progression is about $40-50 \mathrm{~Gy} .{ }^{18}$

In this case report the overall dose was 60Gy. Usually RIS progress within 2 up to 65 years post radiotherapy, with mean period of 15 years. ${ }^{18}$ In this case report the latency interval was 8years. Mark RJ et al..$^{15}$ showed that just $8 \%$ of the patients may survive for 5 years without disease. ${ }^{15}$ The poor outcomes of RIS were confirmed by Patel as well, who showed that the rate of five years survival is worse than other types of H\&N sarcomas. ${ }^{19}$ Many reasons make the prognosis of such sarcomas is really bad, irradiation may lead to some changes in 
the affected area subsequently it delays the diagnosis, involvement of vital neck components prevents complete surgical removal, in addition to the risk of repeating radiotherapy to a previously irradiated field, and the immunosuppressed state of the patient due to the former malignancy and its management. ${ }^{3}$ Since tumor is not responding to chemical agents and re irradiation is not possible, surgical excision is the only valid treatment. However, it's not easy to remove the tumor due to the multifocality nature of the lesion and its infiltration with the surrounding tissue. ${ }^{6}$ In this case report the mass was occupying post cricoid area, pyriform fossa, supraglottis and lateral pharyngeal wall.

\section{Conclusion}

This case report highlights a significant risk of radiation, which needs to be kept in mind during the assessment of malignancy in previously irradiated patients. Clinicians should be aware of long term complications of radiotherapy when they follow up patients with such history.

\section{Acknowledgments}

None.

\section{Conflicts of interest}

The author declares there are no conflicts of interest.

\section{References}

1. Ravichandran R. Has the time come for doing away with cobalt- 60 teletherapy for cancer treatments. $J$ Med Phys. 2009;34(2):63-65.

2. Thiagarajan A, Gopalakrishna N. Radiation-induced sarcomas of the head and neck. World J Clin Oncol. 2014;5(5):973-981.

3. Dilci A, Karakullukçu B, Tan B. Radiation Induced Sarcoma of Hypopharynx: A Case Report. Turk Arch Otolaryngol. 2013;51:131-134.

4. Makimoto Y, Yamamoto $\mathrm{S}$, Takano $\mathrm{H}$, et al. Imaging findings of radiationinduced sarcoma of the head and neck. Br J Radiol. 2007;80(958):790797.

5. Xi M, Liu MZ, Wang HX, et al. Radiation-induced sarcoma in patients with nasopharyngeal carcinoma:a single- institution study. Cancer. 2010;116:5479-5486.
6. Holt GE, Thomson AB, Griffin AM, et al. Multifocality and multifocal postradiation sarcomas. Clin Orthop Relat Res. 2006;450:67-75.

7. Yeang MS, Tay K, Ong WS, et al. Outcomes and prognos- tic factors of post-irradiation and de novo sarcomas of the head and neck: a histologically matched case-control study. Ann Surg Oncol. 2013;20:3066-3075.

8. Huber GF, Matthews TW, Dort JC. Radiation-induced soft tissue sarcomas of the head and neck. J Otolaryngol. 2007;36(2):93-97.

9. Debnam JM, Guha-Thakurta N, Mahfouz YM, et al. Radiationassociated head and neck sarcomas: spectrum of imaging findings. Oral Oncol. 2012;48(2):155-161.

10. Samartzis D, Nishi N, Cologne J, et al. Ionizing radiation exposure and the development of soft-tissue sarcomas in atomic-bomb survivors. $J$ Bone Joint Surg Am. 2013;95:222-229.

11. Rubino C, Shamsaldin A, Lê MG, et al. Radiation dose and risk of soft tissue and bone sarcoma after breast cancer treatment. Breast Cancer Res Treat. 2005;89(3):277-288.

12. Henderson TO, Whitton J, Stovall M, et al. Secondary sarcomas in childhood cancer survivors: a report from the Childhood Cancer Survivor Study. J Natl Cancer Inst. 2007;99(4):300-308.

13. Eeles RA, Fisher C, A'Hern RP, et al. Head and neck sarcomas: prognostic factors and implications for treatment. Br J Cancer. 1993;68(1):201207.

14. Ron E, Modan B, Preston D, et al. Radiation induced skin carcinomas of the head and neck. Radiat Res. 1991;125(3):318-325.

15. Mark RJ, Poen J, Tran LM, et al. Post irradiation sarcomas. Asingleinstitution study and review of the literature. Cancer. 1994;73(10):26532662.

16. Wei Z, Xie Y, Xu J, et al. Radiation induced sarcoma of head and neck: 50years of experience at a single institution in an endemic area of nasopharyngeal carcinoma in China. Med Oncol. 2012;29(2):670-676.

17. Sale KA, Wallace DI, Girod DA, et al. Radiation-induced malignancy of the head and neck. Otolaryngol Head Neck Surg. 2004;131(5):643-645.

18. Murray EM, Werner D, Greeff EA. Postradiation sarcomas: 20cases and a literature review. Int J Radiat Oncol Biol Phys. 1999;45(4):951-961.

19. Patel SG, See AC, Williamson PA, et al. Radiation induced sarcoma of the head and neck. Head Neck. 1999;21(4):346-354. 